\title{
Case of angioid streaks treated with multiple different anti-vascular endothelial growth factor injection
}

\begin{abstract}
Spider-like irregular, bilateral, dark red to gray lines under the retina which serpent from optic disc to border of posterior pole, are classical clinical findings of angioid streaks (AS). Visual alterations commonly seen in middle-aged patients with AS. Calcified and thickened Bruch membrane breaks are responsible clinical manifestations of AS including retinal pigment epithelial and choriocapillary atrophy, intraretinal/subretinal hemorrhage, pigment epithelial detachment, and choroidal neovascular membrane. Laser photocoagulation, macular translocation surgery, transpupillary thermotherapy, and photodynamic therapy were used to perform in treatment of choroidal neovascular membrane seconder to AS, in past. Today, intravitreal anti-vascular endothelial growth factor injection is the most effective and current treatment method because it provides rapid increments in anatomic and functional results and less aggressive than others. But long-term results and sustainability of first reached positive results are controversial. We presented an AS with visual loss in one eye due to choroidal neovascular membrane and treated with intravitreal aflibercept injection after 5 bevacizumab and 3 ranibizumab injection in a total of 6-year followed-up period while visual acuity of the following eye was stable without any treatment. Although the AS have bilateral disease it has different clinical outcomes due to its asymmetric nature. Even the each anti-vascular endothelial growth factor have some effect on treatment of choroidal neovascular membrane further researches are needed that revealed the treatment protocol and their long-term results in case with AS.
\end{abstract}

Keywords: angioid streaks, bevacizumab, ranibizumab, aflibercept
Volume 8 Issue 2 - 2018

\author{
Ayse Gul Kocak Altintas,' Cagri Illhan² \\ 'Associated Professor, University of Health Sciences, Turkey \\ ${ }^{2}$ Hatay State Hospital, Turkey \\ Correspondence: Ayse Gul Kocak Altintas, Associated \\ Professor, University of Health Sciences, Ankara Ulucanlar Eye \\ Education and Research Hospital, Ulucanlar Cad. No: 5906230 \\ Altindag/Ankara/Turkey, Tel + 90-312-3126261, Fax 90-312- \\ 3124827, Email aysegulkaltintas@hotmail.com
}

Received: March 16, 2018| Published: April 23, 2018

\begin{abstract}
Abbreviations: AS, angioid streaks; RPE, retinal pigment epithelium; OCT, optical coherence tomography; FFA, fundus fluorescein angiography; $\mathrm{CNV}$, choroidal neovascular membrane; PED, pigment epithelial detachment; VEGF, vascular endothelial growth factor; BCVA, best corrected visual acuity; RE, right eye; LE, left eye; IOP, intraocular pressures
\end{abstract}

\section{Introduction}

Doyne was descripted angioid streaks (AS) in 1889 as spider-like irregular, bilateral, dark red to gray lines under the retina around optic disk. ${ }^{1}$ Histopathological studies showed that AS represents breaks and crack-like dehiscences in calcified, thickened both collagenous and elastic part of Bruch membrane. Both atrophy and hyperplasia were developed in retinal pigment epithelium (RPE) and destruction of choriocapillary layers were occurred..$^{2}$ Diagnosis of AS is based on presence of the classical clinical appearance on fundus evaluation and optical coherence tomography (OCT). In fundus fluorescein angiography (FFA) hyperfluorescence were observed over the breaks due to RPE atrophy and hypofluorescence associated with RPE hyperplasia. Complications of AS such as choroidal neovascular membrane (CNV), subretinal hemorrhage and pigment epithelial detachment (PED) may cause legal blindness, especially in middleaged patients. While, laser photocoagulation, macular translocation surgery, transpupillary thermotherapy and photodynamic therapy were used to applied in treatment of AS patients with visual loss in last decades, intravitreal anti-vascular endothelial growth factor (VEGF) injection is the most effective and current treatment method for CNV due to $\mathrm{AS}^{3-7}$ In this case report, we presented an AS with visual loss in one eye due to CNV which is followed-up 6-year and treated with multiple different anti-VEGF injection. We aimed to show the risk factor of developing CNV in case with AS, the long-term results of different anti-VEGF therapies, the superiority of them from each other and the effectiveness of switch therapy.

\section{Case report}

We presented a forty-two year old, Caucasian, male patient with AS without any systemic association who has complained of unilateral visual loss. His best corrected visual acuity (BCVA) was 20/20 in right eye (RE) and 20/30 in left eye (LE). Intraocular pressures (IOP) were bilateral $16 \mathrm{mmHg}$ and slit-lamp anterior segment examinations were completely normal. In both eyes' dilated fundus examination, classical AS appearance was seen in posterior pole, in addition, RPE alterations were observed both eyes while minimal hemorrhages was seen in macular region in LE. In FFA, hypofluorescence in angioid streaks borders and widespread hyperfluorescence the in macular region in both eyes. Figure 1 shows colored fundus images and FFA photographs of both eyes (Figure 1). In OCT, there were hyporeflective spaces in outer retinal layers and hyperreflective band between outer retina and RPE in both eyes. When comparing the findings of two eyes, they were seen that the lesion was parafoveal and fovea relatively spared in right eye and BCVA was 20/20 while the lesion was more subfoveal in left eye and BCVA was decreased. 


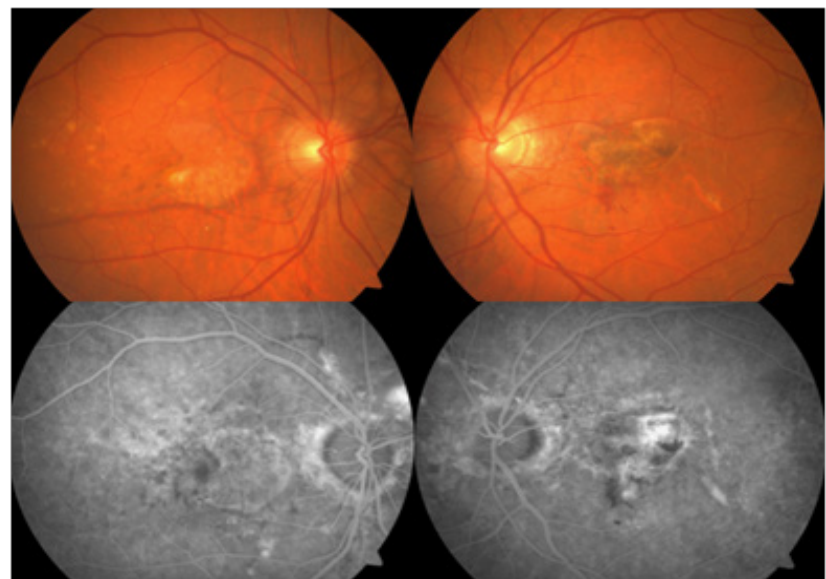

Figure I Colored fundus images and fundus fluorescein photographs of both eyes.

Intravitreal anti-VEGF injection was planned for left eye of patient. Repeating intravitreal bevacizumab injections were performed 5-time in 2-year period according to his BCVA, clinical and OCT findings. After the first bevacizumab injection, BCVA increased to 20/25 but 2-month later it suddenly decreased in 20/70 due to submacular hemorrhage and second intravitreal bevacizumab injection was applied. One month later BCVA increased to 20/25 again. Three months later his BCVA was still 20/25 but he had complained for dysmorphopsia. He had been treating with bevacizumab according to patient's clinical and OCT findings. Patient's functional response of bevacizumab therapy was significantly unstable and subjective symptoms were gradually increased. After 2-year follow-up, even the BCVA was not significantly decreased, due to recurrence of macular fluid and increment of visual symptoms, intravitreal ranibizumab injection was planned. After 3 consecutive ranibizumab injection were performed in one year period, BCVA was remained stable which was $20 / 30$.

One year later, the patient presented with 20/200 BCVA without any trauma. Severe submacular hemorrhage observed on clinically and $\mathrm{CNV}$ was found in OCT and FFA evaluations. Hence, intravitreal ranibizumab injection switched to aflibercept therapy. Consecutive 3 injections of aflibercept were performed in one month interval. BCVA was gradually increased to $20 / 60$, submacular hemorrhage was disappeared, macular thickness on OCT due to hemorrhage was disappeared. BCVA was remained as 20/60 even with development of slight lens opacification for one year follow-up after last intravitreal injection. In final OCT, hyporeflective spaces were resolved and legacy outer retinal tubulation was detected which can be seen in natural course of AS (Figure 2). ${ }^{8}$
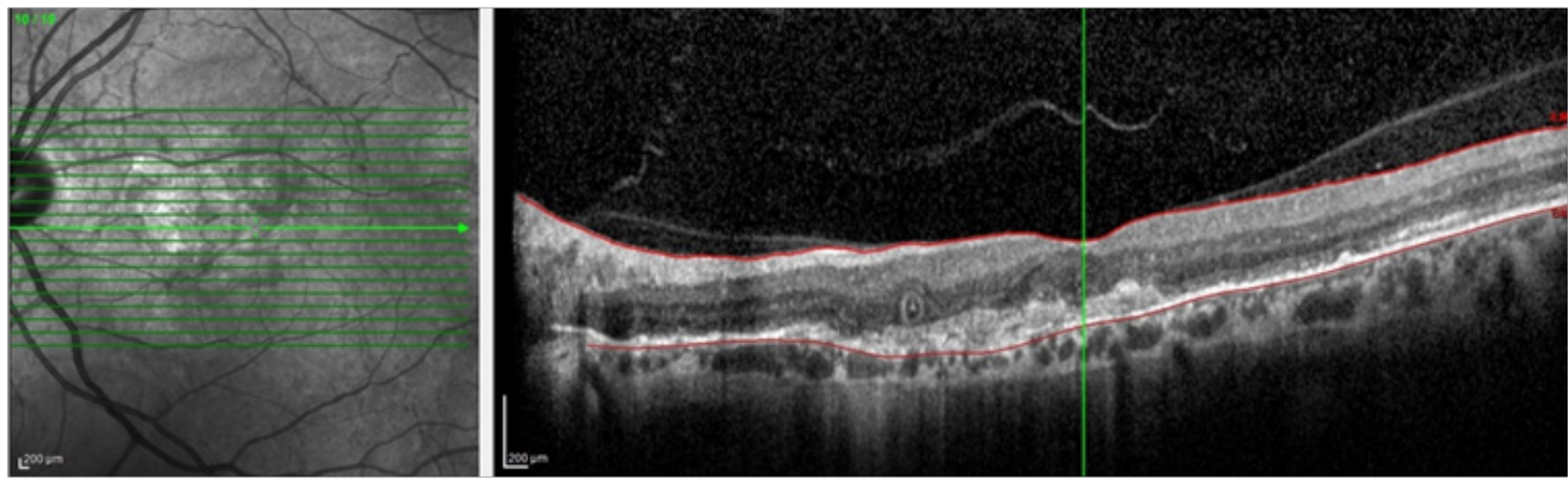

Figure 2 Outer retinal tubulation, resolved hyporeflectivity and increased outer retinal hyperreflectivity in left eye's optical coherence tomography

\section{Discussion}

Intravitreal anti-VEGF injection provides the most rapid visual recovery in $\mathrm{CNV}$ and the least destructive method for retinal structure. So, in today anti-VEGF therapy is seemed as the first treatment choice in $\mathrm{CNV}$ with different etiologies. $\mathrm{CNV}$ is responsible the most majority of low vision in cases with AS. ${ }^{9}$ Subfoveal lesions have greater mischance in regard of visual loss. We planned anti-VEGF therapy for left eye of patient because it has subfoveal $\mathrm{CNV}$ due to subfoveal breaks in Bruch membrane even the visual acuity was not deeply affected initially, on the other hand, the lesions in right eye was stable during the all follow-up years and visual acuity did not change, where the breaks were occurred parafoveal region.

Alagoz et al. ${ }^{10}$ treated 23 eyes with $\mathrm{CNV}$ due to AS, with mean 5.1 intravitreal bevacizumab injection in mean 23-month follow-up. They stated that visual acuity was increased or preserved in $65.2 \%$ of eyes, however CNV was stable in only 39\% eyes in last visit. This article studied the effect of intravitreal bevacizumab injection in AS, has the largest sample size in literature and it shows that relatively positive and low-sustainable effects of this therapy in medium/longterm. ${ }^{10}$ In Lekha et al. ${ }^{11}$ study showed that recurrence was observed in $73.33 \%$ eyes after 5.60 bevacizumab injection over 57.33 months follow-up. ${ }^{11}$ They concluded that more effective options are needed to reduce risk of recurrence. ${ }^{11}$ Similarly, we observed sharp changes both significant decrease and increase in patient's visual acuity during the 5 bevacizumab injections in 2.5 years follow-up and first achieved positive anatomic and functional responses could not preserved, patient had complained of severe image distortion.

Tilleul et al. ${ }^{12}$ treated 35 eyes of 27 patients with ranibizumab. They stated that end of the 48 months follow-up period, BCVA was improved or stabilized in $62.9 \%$ eyes and macular thickness was stabilized or decreased in $45.7 \%$ eyes. In addition, no further leakage was observed in $77.1 \%$ eyes on FFA at the last visit. ${ }^{12}$ They reported that in the light of this values, when compared the effectiveness of bevacizumab and ranibizumab, intravitreal ranibizumab injection seems more effective in long-term results of $\mathrm{CNV}$ due to AS. We performed 3 successive intravitreal injection of ranibizumab without need of any other 
treatment in following 2 years period after 5 bevacizumab injections in 2.5 years which is the nearly same evaluation period. Visual acuity was stable and complained of dysmorhopsia was totally disappeared and no recurrence was observed both clinically and in OCT findings with the less injections comparing to previous bevacizumab treatment. We observed that effect of IV ranibizumab injection last longer than bevacizumab.

Aflibercept is a new developed anti-VEGF agent that inhibits all isoforms of VEGF-A, VEGF-B and placental growth factor. ${ }^{13}$ Currently there are no any clinical trial in literature investigated the efficacy of aflibercept in CNV secondary to AS and only few cases reported. Esen et al. ${ }^{14}$ reported the clinical reports of switching from ranibizumab to aflibercept. After 3 intravitreal aflibercept injections at one month intervals for both of two eyes, visual acuity improved, intraretinal/subretinal fluid resolved, and central macular thickness reduced. ${ }^{14}$ We performed successively 3 aflibercept injections as another opinion for our patient after significant decreased in visual acuity due to intensive recently developed submacular hemorrhage with the recurrence of $\mathrm{CNV}$ and we reached the favorable anatomic and acceptable functional results without any recurrence. While other eye with the creaks sparing of the fovea was stable in terms of visual acuity in these 6-year period.

\section{Conclusion}

AS may occur with significantly asymmetric involvement in each eyes of the same patient. Eyes with creaks of Bruch membrane was located under the fovea has high risk about visual loss due to CNV. Intravitreal anti-VEGF injection is most current treatment modality and even the favorable results are obtained with both ranibizumab and aflibercept, since the underlying etiological factor are persisted recurrence may occur any time. Therefore close follow-up in long term and prompt treatment of patient have high risk of recurrence is essential. At the same time, further researches are needed that revealed the long-term results and treatment regime of each antiVEGF application in AS.

\section{Acknowledgements}

None.

\section{Conflict of interest}

The author declares there is no conflict of interest.

\section{References}

1. Doyne RW. Choroidal and retinal changes. The result of blows on the eyes. Trans Ophthalmol Sok UK. 1889;9:128.

2. Domke H, Tost M. On the histology of "Angioid Streaks". Klin Monbl Augenheilkd. 1964;145:18-29.

3. Gliem M, Finger RP, Fimmers R, et al. Treatment of choroidal neovascularization due to angioid streaks: A comprehensive review. Retina. 2013;33(7):1300-1314.

4. Gelisken O, Hendrikse F, Deutman AF. A long-term follow-up study of laser coagula- tion of neovascular membranes in angioid streaks. $\mathrm{Am} \mathrm{J}$ Ophthalmol. 1988;105(3):299-303.

5. Ehlers JP, Maldonado R, Sarin $\mathrm{N}$ et al. Treatment of non-age-related macular de- generation submacular diseases with macular translocation surgery. Retina. 2011;31(7):1337-1346.

6. Ozdek S, Bozan E, Gürelik G, et al. Transpupillary thermotherapy for the treatment of choroidal neovascularization secondary to angioid streaks. Can J Ophthalmol. 2007;42(1):95-100.

7. Lee JM, Nam WH, Kim HK. Photodynamic therapy with verteporfin for choroidal neo- vascularization in patients with angioid streaks. Korean $J$ Ophthalmol. 2007;21(3):142-145.

8. Giachetti Filho RG, Zacharias LC, Monteiro TV, et al. Prevalence of outer retinal tubulation in eyes with choroidal neovascularization. Int $J$ Retina Vitreous. 2016;2:6.

9. Pece A, Avanza P, Galli L, et al. Laser photocoagulation of choroidal neovascularization in angioid streaks. Retina. 1997;17(1):12-16.

10. Alagöz C, Alagöz N, Özkaya A, et al. Intravitreal bevacizumab in the treatment of choroidal neovascular membrane due to angioid streaks. Retina. 2015;35(10):2001-2010.

11. Lekha T, Prasad HN, Sarwate RN, et al. Intravitreal Bevacizumab for Choroidal Neovascularization Associated with Angioid Streaks: Longterm Results. Middle East Afr J Ophthalmol. 2017;24(3):136-142.

12. Tilleul J, Mimoun G, Querques G, et al. Intravitreal ranibizumab for choroidal neovascularization in angioid streaks: Four-Year Follow-up. Retina. 2016;36(3):483-491.

13. Papadopoulos N, Martin J, Ruan Q, et al. Binding and neutralization of vascular endothelial growth factor (VEGF) and related ligands by VEGF Trap ranibizumab and bevacizumab. Angiogenesis. 2012;15(2):171-185.

14. Esen E, Sizmaz S, Demircan N. Intravitreal aflibercept for management of subfoveal choroidal neovascularization secondary to angioidstreaks. Indian J Ophthalmol. 2015;63(7):616-618. 